\title{
Ex Vivo Nicotine Stimulation Augments the Efficacy of Human Peripheral Blood Mononuclear Cell-Derived Dendritic Cell Vaccination via Activating Akt-S6 Pathway
}

\author{
Yan Yan Wang, ${ }_{1}^{1}$ Yi Wen Yang, ${ }^{1}$ Xiang You, ${ }^{1}$ Xiao Qian Deng, ${ }^{1}$ Chun Fang Hu, ${ }^{1}$ Cong Zhu, \\ Jun Yao Wang, ${ }^{1}$ Jiao Jiao Gu, ${ }^{1}$ Yi Nan Wang, ${ }^{1}$ Qing Li, ${ }^{1}$ and Feng Guang Gao ${ }^{1,2}$ \\ ${ }^{1}$ Department of Immunology, Basic Medicine Science, Medical College, Xiamen University, Xiamen 361102, China \\ ${ }^{2}$ The State Key Laboratory for Oncogenes and Related Genes, Shanghai Jiao Tong University, Shanghai 200032, China \\ Correspondence should be addressed to Qing Li; sunnymaylq@hotmail.com and Feng Guang Gao; gfengguang@xmu.edu.cn
}

Received 7 April 2015; Revised 10 June 2015; Accepted 18 June 2015

Academic Editor: Consuelo Amantini

Copyright (c) 2015 Yan Yan Wang et al. This is an open access article distributed under the Creative Commons Attribution License, which permits unrestricted use, distribution, and reproduction in any medium, provided the original work is properly cited.

Our previous studies showed that $\alpha 7$ nicotinic acetylcholine receptor (nAchR) agonist nicotine has stimulatory effects on murine bone marrow-derived semimature DCs, but the effect of nicotine on peripheral blood mononuclear cell- (PBMC-) derived human semimature dendritic cells (hu-imDCs) is still to be clarified. In the present study, hu-imDCs (cultured 4 days) were conferred with ex vivo lower dose nicotine stimulation and the effect of nicotine on surface molecules expression, the ability of crosspresentation, DCs-mediated PBMC priming, and activated signaling pathways were determined. We could demonstrate that the treatment with nicotine resulted in increased surface molecules expression, enhanced hu-imDCs-mediated PBMC proliferation, upregulated release of IL-12 in the supernatant of cocultured DCs-PBMC, and augmented phosphorylation of Akt and ribosomal protein S6. Nicotine associated with traces of LPS efficiently enhanced endosomal translocation of internalized ovalbumin (OVA) and increased TAP-OVA colocalization. Importantly, the upregulation of nicotine-increased surface molecules upregulation was significantly abrogated by the inhibition of Akt kinase. These findings demonstrate that ex vivo nicotine stimulation augments huimDCs surface molecules expression via Akt-S6 pathway, combined with increased Ag-presentation result in augmented efficacy of DCs-mediated PBMC proliferation and Th1 polarization.

\section{Introduction}

Dendritic cells (DCs) are professional antigen-presenting cells (APCs) that recognize extracellular antigens in the peripheral tissue. On recognition of microbial substances, DCs migrate toward the draining lymph node, where they can induce antigen-specific T-cell priming and reveal therapeutic and protective antitumor immunity $[1,2]$. In previous studies, we have demonstrated that ex vivo nicotine stimulation has stimulatory effects on murine bone marrow-derived semimature DCs (imDCs), which reveal efficient upregulation of surface molecules through $\alpha 7$ nicotinic acetylcholine receptor (nAchR) [3-6]. Further studies showed that the treatment with nicotine increases surface molecules expression via PI3K-Akt pathway and nicotine-treated murine imDCs has antitumor effects in both Lewis lung cancer and hepatocellular carcinoma $[4,5]$. Although several groups have documented that nicotine has positive effects in the treatment of neurodegenerative diseases, ulcerative colitis, and Tourette syndrome [7-9], it is the first time of our studies demonstrating that nicotine-treated imDCs have preventive and therapeutic effects on murine tumor formation. However, the effect of ex vivo nicotine stimulation on human peripheral blood mononuclear cells- (PBMC-) derived imDCs (huimDCs) is still uncertain.

For T-cell activation, internalized antigens are degraded, and the resulting peptides are loaded to MHC molecules and transported to plasma membrane, where these complexes are recognized by antigen-specific $\mathrm{T}$ cells. While peptides which resulted from soluble antigen can be loaded to MHC class II 
molecules, it can also be loaded to MHC class I molecules, a process termed cross-presentation $[10,11]$. Efficient crosspresentation requires antigen uptake-mediated entrance of antigen into early endosome [12], which was mediated by mannose receptor [13]. Meanwhile, the critical role of proteasome in generating epitopes and transporting internalized antigen across the endosomal membrane into cytoplasm was also documented [14]. Although previous studies revealed that ex vivo nicotine stimulation increases antigen internalization and promotes imDCs' cross-presentation [3-6], the exact effect of endosomal translocation of internalized antigen in nicotine-increased cross-presentation is still uncertain, nevertheless the effect of nicotine on the transporting internalized antigen to cytoplasm.

In the present study, hu-imDCs (cultured 4 days) were conferred with ex vivo lower dose nicotine stimulation and the effect of nicotine on surface molecules expression, the ability of cross-presentation, DCs-mediated PBMC priming, and activated signaling pathways were determined. We demonstrate that ex vivo nicotine stimulation results in increased surface molecules expression, enhanced hu-imDCs-mediated PBMC proliferation, upregulated the release of IL-12, increased the percentage of CD8/IFNgamma double positive cell in cocultured DCs-PBMC, and augmented phosphorylation of Akt and ribosomal protein S6. Nicotine associated with traces of LPS efficiently enhanced endosomal translocation of internalized ovalbumin (OVA) and increased TAP-OVA colocalization. Importantly, nicotine-increased upregulation of surface molecules was significantly abrogated by the inhibition of Akt kinase. These findings demonstrate that ex vivo nicotine stimulation augments hu-imDCs surface molecules expression via AktS6 pathway, combined with increased Ag-presentation result in augmented efficacy of DCs-mediated PBMC proliferation and Th1 polarization.

\section{Materials and Methods}

2.1. Reagents. Reagents were purchased from the following companies: (-)-nicotine (N3876), lipopolysaccharide (LPS), $\alpha$-bungarotoxin-tetramethylrhodamine from Bungarus multicinctus (Formosan Banded Krait) (T0195), and tubocurarine chloride were obtained from Sigma-Aldrich (St. Louis, MO, USA). Recombinant human GM-CSF and IL-4 were obtained from PeproTech (Rocky Hill, NJ, USA). Endotoxinfree EndoGrade-ovalbumin (OVA) was purchased from Hyglos GmbH (Regensburg, Germany). PI3K inhibitor LY294002 and Akt inhibitor Wortmannin were from Cayman Chemical (Ann Arbor, MI, USA). Purified anti-chicken ovalbumin (OVA) (Clone TOSGAA1) was obtained from Biolegend (San Diego, CA). Antibodies to EEA1, Rab7, and TAP were from Cell Signaling Technology (Beverly, MA, USA). RPMI1640 medium and fetal bovine serum (FBS) were purchased from HyClone (Logan, UT, USA). BD Phosflow antibodies to phospho-p38 (p-T180/pY182) (Clone 36/p38), phosphoErk1/2 (p-T202/pY204) (Clone 20A), phospho-Akt (pS473) (Clone M89-61), phospho-S6 (pS235/pS236) (Clone N7-548), and human IL-12 ELISA Kit were from BD Biosciences (San
Jose, CA, USA). BrdU Cell Proliferation Kit was obtained from Roche (Roche Diagnostics GmbH, Germany). Fluorescence conjugated antibodies to human CD80, CD86, 4$1 \mathrm{BBL}, \mathrm{CD} 8$, IFN- $\gamma$, MHC class I, and MHC class II molecules were obtained from eBioscience (San Diego, USA). Mounting medium for fluorescence with DAPI was obtained from Vector Laboratories, Inc. (Burlingame, CA, USA). Human PBMC isolation reagent was from Haoyang Biological Manufacture Co., Ltd. (Tianjin, China). Rab5, goat anti-mouse IgG and donkey anti-goat IgG secondary antibodies were from Abcam (New Territories, HK).

2.2. Generation of Human PBMC-Derived imDCs. Briefly, human PBMC was prepared from the donor's blood by gradient density centrifugation using PBMC isolation reagents and cultured at a density of $1 \times 10^{6}$ cells $/ \mathrm{mL}$ in RPMI 1640 medium in the presence of $100 \mathrm{ng} / \mathrm{mL}$ recombinant human GM-CSF and $100 \mathrm{ng} / \mathrm{mL}$ IL-4. Nonadherent cells were gently washed out with PBS on day 4; the remaining loosely adherent clusters were used as hu-imDCs. Cells were synchronized by serum starvation (in RPMI 1640 with $0.5 \%$ FBS) for $4 \sim 6 \mathrm{~h}$ before the further treatment. This study was conducted with the understanding and the consent of all the blood donors and was approved by the Institutional Review Board for Human Subjects at the Medical College of Xiamen University.

2.3. Human imDCs Treatment. To determine the effect of ex vivo nicotine stimulation on surface molecules expression, hu-imDCs were exposed to nicotine $\left(10^{-7} \mathrm{~mol} / \mathrm{L}\right)$ for 12 to $15 \mathrm{~h}$ after cell synchronization by $0.5 \%$ serum starvation. The pretreatment with $2 \mu \mathrm{g} / \mathrm{mL} \alpha$-bungarotoxin or $\left(4 \times 10^{-5} \mathrm{~mol} / \mathrm{L}\right)$ tubocurarine chloride $2 \mathrm{~h}$ prior to nicotine stimulation was conducted to investigate the role of $\alpha 7 \mathrm{nAchR}$ in nicotineincreased DCs' function. To elucidate the mechanism of nicotine-increased surface molecules expression, hu-imDCs were conferred with LY294002 $(10 \mu \mathrm{mol} / \mathrm{L})$ or Wortmannin $(10 \mu \mathrm{mol} / \mathrm{L}) 2 \mathrm{~h}$ prior to nicotine $\left(10^{-7} \mathrm{~mol} / \mathrm{L}\right)$ exposure.

2.4. Flow Cytometric Measurement for Surface Molecules. The expression of surface molecules in hu-imDCs was determined by flow cytometry. Briefly, hu-imDCs were preincubated with $0.5 \mu \mathrm{g}$ CD16/CD32 antibodies for $10 \mathrm{~min}$. Then, aliquot cell suspension was stained with combined primary antibody at a concentration of $1 \mu \mathrm{g}$ per $1 \times 10^{6}$ cells. Staining was performed on ice for $30 \mathrm{~min}$ and cells were washed with ice-cold PBS, containing $0.1 \% \mathrm{NaN} 3$ and $0.5 \%$ BSA. Flow cytometry was done with FACSCalibur and data were analyzed with CellQuest software.

2.5. Flow Cytometric Measurement for Intracellular Signaling Molecules. To determine the effect of nicotine on kinases phosphorylation, hu-imDCs were collected by trypsination and treated with nicotine $\left(10^{-7} \mathrm{~mol} / \mathrm{L}\right)$ for $15 \mathrm{~min}$. The phosphorylation of related kinase was determined by BD Phosflow. Briefly, at the end of nicotine treatment, the cells were immediately mixed with warmed BD Phosflow Fix Buffer I and incubated at $37^{\circ} \mathrm{C}$ for $10 \mathrm{~min}$. Then, the cells were washed with BD Pharmingen Stain Buffer and permeabilized 
by incubation with cold BD Phosflow Perm Buffer III for 30 min on ice. After complete washes, the cells were stained with BD Phosflow antibodies and flow cytometry was done with FACSCalibur and data were analyzed with CellQuest software.

2.6. Confocal Microscope Analyses. The effect of nicotine on the endosomal translocation of antigen and the complex formation of ovalbumin-derived peptide-MHC class I and II molecules were investigated by confocal microscope analyses. Briefly, hu-imDCs were exposed to nicotine $\left(10^{-7} \mathrm{~mol} / \mathrm{L}\right)$ for $12 \sim 15 \mathrm{~h}$. Then, the cells were conferred with endotoxinfree EndoGrade-ovalbumin $(50 \mu \mathrm{g} / \mathrm{mL}) 60 \mathrm{~min}$ pulse with or without short period $(20 \mathrm{~min})$ LPS $(1 \mathrm{ng} / \mathrm{mL})$ stimulation. After that, the DCs were fixed and permeabilized in $100 \%$ methanol for $15 \mathrm{~min}$, washed with PBS, and blocked with $10 \%$ nonfat milk for $3 \mathrm{~h}$. Primary antibodies were incubated in a humid chamber overnight at $4^{\circ} \mathrm{C}$. Finally, fluorescenceconjugated secondary antibodies were incubated for $1 \mathrm{~h}$ at $37^{\circ} \mathrm{C}$. DAPI counterstaining was performed to visualize cell nuclei. The cell was washed three times in each step to remove nonbinding substance and images were recorded by a confocal fluorescence microscope at the wavelength of $488 \mathrm{~nm}$.

2.7. Ag-Specific PBMC Proliferation and CTL Priming Assay. Briefly, hu-imDCs (cultured for $4 \mathrm{~d}$ ) were pretreated with $\alpha$-bungarotoxin $(2 \mu \mathrm{g} / \mathrm{mL})$ or tubocurarine chloride $(4 \times$ $\left.10^{-5} \mathrm{~mol} / \mathrm{L}\right) 2 \mathrm{~h}$ prior to nicotine treatment. Then, the DCs were conferred with $3 \mathrm{~h}$ endotoxin-free EndoGradeovalbumin $(50 \mu \mathrm{g} / \mathrm{mL})$ pulse with $60 \mathrm{~min}$ LPS $(1 \mathrm{ng} / \mathrm{mL})$ stimulation. Responder cells were prepared from PBMC of the same donor. Stimulator cells were mixed with responders at a ratio of $1: 1$. After $5 \mathrm{~d}$ of coculture, Ag-specific PBMC proliferation was determined by BrdU cell proliferation assay. To determine CTL priming, the proliferated PBMC cells were performed intracellular IFN-gamma and CD8 positive flow cytometric analyses.

2.8. Quantification of IL-12 Production. The release of IL-12 in the supernatant of MLR was determined by ELISA according to the standard procedure. Briefly, plates were treated with coating antibody at $4^{\circ} \mathrm{C}$ overnight, washed with PBS, and blocked with assay buffer at room temperature for $2 \mathrm{~h}$. The blocked plates were washed twice with thorough aspiration of microwell contents between washes. After the last wash step, empty wells and tap microwell strip to remove excess wash buffer. Samples of assay buffer, biotin-conjugated detector antibodies were added to microwells and the plates were incubated at room temperature for $2 \mathrm{~h}$. After incubation, plates were washed 5 times and added with streptavidinHRP. After $1 \mathrm{~h}$ of incubation at room temperature, plates were washed and TMB substrate solution was added to all wells for color formation. Plates were incubated at room temperature and stop solution was added to stop the enzyme reaction at appropriate time. Absorbance of each microwell was read using $450 \mathrm{~nm}$ as primary wave length and $620 \mathrm{~nm}$ as reference wavelength, respectively.
2.9. Western Blot. For analysis of Akt and S6 phosphorylation regulated by nicotine on hu-imDCs, proteins were obtained in lysis buffer. Protein lysates $(30 \mu \mathrm{g} / \mathrm{mL})$ were electrophoresed on 10\% SDS-PAGE gels, transferred to PVDF membranes, and blotted with phospho-Akt and phospho-S6 antibodies, followed by anti-mouse horseradish peroxidase and detection by chemiluminescence ECL. As loading controls, antibody against $\beta$-actin was used.

2.10. Statistical Analysis. All data were expressed as mean and standard error means. Statistical significance was tested using the Student $t$-test or one-way ANOVA with postNewman-Keuls test. Statistical differences were considered to be significant if $P<0.05$.

\section{Results}

3.1. Nicotine Upregulates Surface Molecules Expression in Human Semimature DCs. Human immature monocytederived DCs are commonly generated by culturing adherent peripheral monocytes with GM-CSF and IL- 4 for up to 6 days [15-17]. Meanwhile, in a 4-day culture system, murine semimature DCs could differentiate into a regulatory DCs subset by splenic stromal cells [18] and have potential antitumor effects [3-6]. To explore the effect of nicotine on DCs' maturation and viability, hu-imDCs (cultured 4 days) were conferred with ex vivo nicotine stimulation. Consistent with murine semimature DCs' results $[19,20], 10^{-7} \mathrm{~mol} / \mathrm{L}$ nicotine has no effect on cell viability of hu-imDCs (see Supplementary Figure 1 in Supplementary Material available online at http://dx.doi.org/10.1155/2015/741487). While $83.43 \%$ vehicletreated hu-imDCs expressed human DCs specific marker CDla, the treatment with nicotine increased the level of CDla to about $150 \%$ (Supplementary Figure 2a). The analyses of CD1lc expression also achieved the similar results (Supplementary Figure $2 \mathrm{~b}$ ). Considering ex vivo lower dose nicotine stimulation promotes the development of mouse semimature DCs $[20,21]$, the 4-day culture system might be a specific condition for the effect of lower dose nicotine stimulation on hu-imDCs.

MHC class I and II molecules are the components of antigenic peptide-MHC complex for antigen presentation [10-14]. While CD80/CD86 are important costimulatory molecules in T-cell-APC interaction [2, 22], 4-1BBL, which sends signals to $4-1 \mathrm{BB}$-expressing cells $[23,24]$, was found to play critical roles in preventing activation-induced cell death $[25,26]$. To explore the exact effect of nicotine stimulation on the expression of surface molecules, hu-imDCs were conferred with nicotine exposure and the expression of CD80, 4-1BBL, and MHC class I and II molecules was determined by flow cytometry. Compared with vehicletreated cells, the treatment with nicotine obviously increased the expression of CD80, 4-1BBL, revealing about $177 \%$ and $131 \%$ upregulation, respectively (Figures $1(\mathrm{a})$ and $1(\mathrm{~b})$ ). The determination of MHC class I and II molecules also achieved similar results (Figures 1(c) and 1(d)). Interestingly, when dot plot of flow cytometry was used to investigate the effect of nicotine on DCs maturation, the treatment with nicotine not 


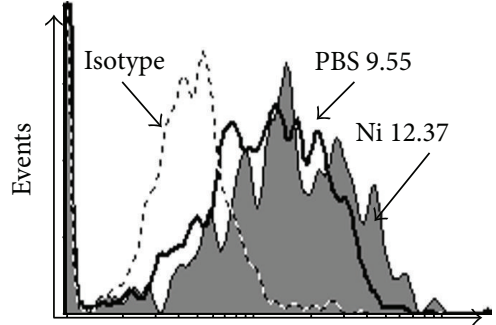

CD80-fluorescence

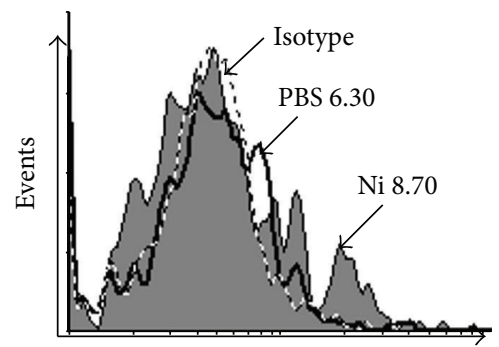

4-1BBL-fluorescence

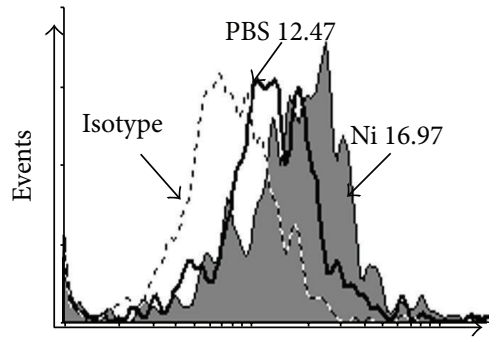

MHC I-fluorescence

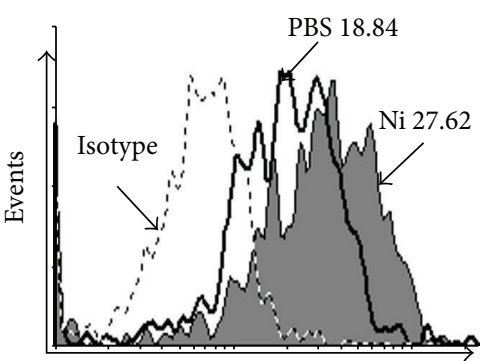

MHC II-fluorescence

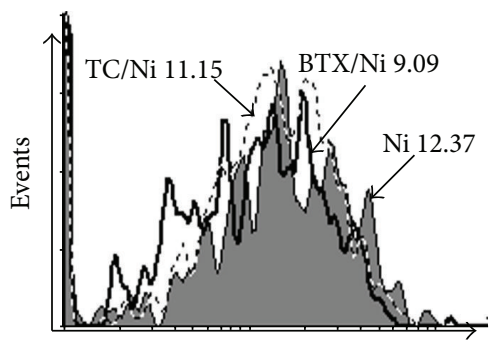

CD80-fluorescence

(a)

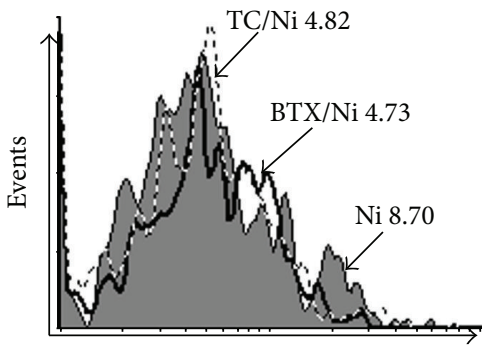

4-1BBL-fluorescence

(b)

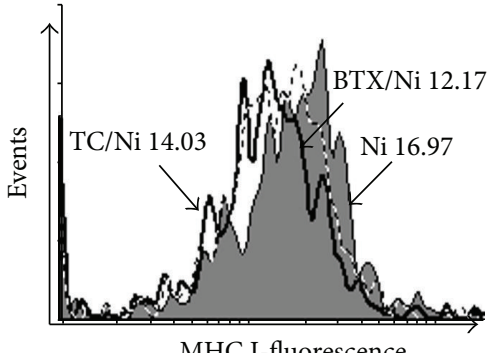

(c)

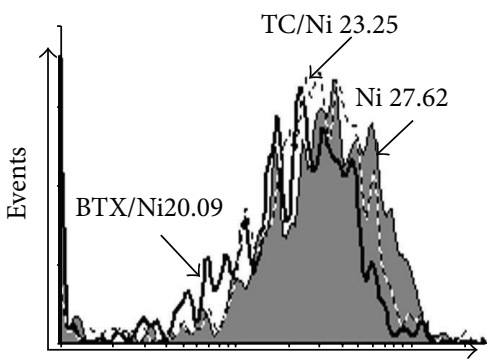

MHC II-fluorescence
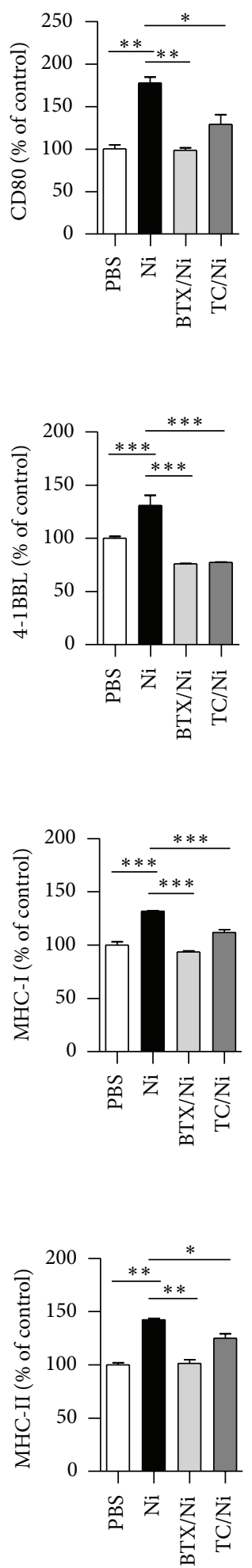

(d)

Figure 1: Nicotine upregulates surface molecules expression in human semimature DCs. (a-d) DCs derived from human PBMC with $100 \mathrm{ng} / \mathrm{mL}$ recombinant human GM-CSF and IL- 4 were conferred with $\alpha$-bungarotoxin $(2 \mu \mathrm{g} / \mathrm{mL})$ or tubocurarine chloride $\left(4 \times 10^{-5} \mathrm{~mol} / \mathrm{L}\right)$ $2 \mathrm{~h}$ prior to nicotine $\left(10^{-7} \mathrm{~mol} / \mathrm{L}\right) 12 \sim 15 \mathrm{~h}$ stimulation. The effect of nicotine on the expression of CD80 (a), 4-1BBL (b), MHC class I (c), and MHC class II (d) molecules was determined by flow cytometry. Numbers in histogram indicated mean fluorescence intensity (MFI) of test samples. Data were given as mean \pm SEM, one-way ANOVA with post-Newman-Keuls test. ${ }^{*} P<0.05,{ }^{* *} P<0.01$, and ${ }^{* * *} P<0.001$. One representative from 3 independent experiments is shown. Ni: nicotine; BTX: $\alpha$-bungarotoxin; and TC: tubocurarine chloride. 
only increased CDla-CD11c double positive DCs' percentage (Supplementary Figure 3a) but also augmented MHC class IICD86 double positive cell population (Supplementary Figure $3 b)$. These data indicate that ex vivo nicotine stimulation not only increases the expression of CD80 and 4-1BBL but also upregulates the expression of MHC class I and II molecules in hu-imDCs.

$\alpha 7 \mathrm{nAchR}$, which is mainly expressed in DCs, is involved in nicotine-augmented expression of surface molecules in murine semimature DCs [19]. To investigate the potential roles of $\alpha 7 \mathrm{nAchR}$ in nicotine-increased surface molecules expression, hu-imDCs were treated with $\alpha 7 \mathrm{nAchR}$ specific $\alpha$-bungarotoxin or nonspecific antagonist tubocurarine chloride prior to nicotine stimulation. Despite the expression of CD80, 4-1BBL, and MHC class I and II molecules obviously upregulated by the treatment with nicotine, the usage of $\alpha$ bungarotoxin and tubocurarine chloride efficiently abrogated nicotine's effect on surface molecules expression (Figure 1), indicating that nicotine increasing surface molecules expression is $\alpha 7 \mathrm{nAchR}$-dependent.

3.2. Ex Vivo Nicotine Stimulation Augments Semimature DCsMediated PBMC Proliferation and Promotes the Release of $I L-12$ in the Supernatant of Cocultured DCs-PBMC. Our previous studies showed that the treatment with lower dose nicotine promotes mouse semimature DCs-mediated cross priming [3-6]. Since ex vivo nicotine stimulation increases the expression of surface molecules in hu-imDCs (Figure 1, Supplementary Figure 3), we accessed the exact effect of nicotine stimulation on hu-imDCs-mediated PBMC proliferation and Th1 polarization by cocultured ex vivo nicotinestimulated, ovalbumin-loaded hu-imDCs with PBMC. As endotoxins increase antigen processing in both MHC class II-restricted antigen presentation and intracellular mechanisms of cross-presentation $[27,28]$, we mimic the ordinary antigen by giving traces of LPS $(1 \mathrm{ng} / \mathrm{mL})$ to endotoxin-free EndoGrade-ovalbumin. Compared with controls, while the loading with endotoxin-free ovalbumin and LPS promotes hu-imDCs-mediated PBMC proliferation, ex vivo nicotine stimulation efficiently augmented the ability of hu-imDCsdependent PBMC proliferation $(P<0.001)$. The pretreatment with $\alpha$-bungarotoxin or tubocurarine chloride dramatically abolished nicotine-increased hu-imDCs mediated PBMC proliferation, which revealed about $26.65 \%$ and $43.26 \%$ inhibitory rates, respectively, (Figure 2(a)), indicating that nicotine exposure-increased hu-imDCs-mediated PBMC proliferation is $\alpha 7 \mathrm{nAchR}$-dependent.

IL-12, an indicator of antigen-specific CTL priming, can polarize Th0 to Thl transition and subsequently induce antivirus and antitumor immune responses [29]. Since the treatment with nicotine increased hu-imDCs-mediated PBMC proliferation, we further explored if such stimulation could polarize Th0 to Th1 transition by the IL-12 determination in the supernatant of cocultured DCs-PBMC. Compared with controls, the pulse of endotoxin-free ovalbumin with traces of LPS obviously augmented IL-12 release in the supernatant. Ex vivo nicotine stimulation efficiently increased IL-12 release to about $167 \%$. The pretreatment with $\alpha$-bungarotoxin or tubocurarine chloride obviously abolished nicotine-increased IL-12 secretion with 58\% 70\% inhibitory rate (Figure 2(b)). Flow cytometric measurement of intracellular IFN-gamma showed that the percentage of double positive cell of IFN-gamma and CD8 was also increased by the treatment with ex vivo nicotine stimulation (Figure 2(c)). These data indicate that the treatment with nicotine facilitates Thl polarization and CTL priming in DCsmediated PBMC proliferation.

3.3. The Exposure of Traces of LPS Increases the Endosomal Translocation of Antigen and TAP-OVA-MHC Class I Molecules Complex Formation in Human Semimature DCs. Mannose receptor, which is expressed in human DCs, uptakes antigen and determines subcellular antigen localization [31]. The localization of model antigen ovalbumin in early endosomal compartment [13] did not further mature early endosome into lysosomes but promote antigen for crosspresentation [12]. Meanwhile, microbial products such as LPS were demonstrated to trigger a program of DCs maturation which enables DCs to activate T cells [14]. To elucidate the role of LPS in nicotine-increased antigen early endosomal translocation, we pulsed hu-imDCs briefly $(60 \mathrm{~min})$ with endotoxin-free EndoGrade-ovalbumin, concurrently either with short period (20 $\mathrm{min}$ ) LPS $(1 \mathrm{ng} / \mathrm{mL}$ ) stimulation or for the same length of time but without LPS exposure (Figure 3). We assessed endosomal translocation of antigen by ovalbumin and EEA1/Rab7 antibodies staining. Coadministration of endotoxin-free EndoGrade-ovalbumin with short period LPS exposure resulted in significantly enhanced translocation of ovalbumin to endosomal compartment (Figure 3). As efficient cross-presentation requires the entrance of antigen into a specific intracellular pathway [12], the increased endosomal translocation of ovalbumin by lower dose LPS indicates that nicotine-increased cross-presentation needs mannose receptor-mediated endosomal translocation of internalized antigen.

While antigens internalization via mannose receptormediated endocytosis was routed into a distinct murine endosomal subset $[10,11]$, these endosomal subsets could reimport proteasome-derived peptides into the same endosomal compartment by LPS-inducing endosomal translocation of TAP, thereafter loading these peptides onto MHC I molecules [12]. To explore the role of LPS-inducing signaling in TAP-mediated antigenic transport, we assessed the translocation of antigen, TAP, and MHC class I and II molecules in lower dose LPS $(1 \mathrm{ng} / \mathrm{mL})$ presenting condition with related antibodies staining. Compared with nicotinetreated hu-imDCs, the signaling induced by LPS administration resulted in significantly enhanced translocation of OVA-TAP and OVA-MHC class I molecules (Figure 4). The colocalization of TAP-MHC class I molecules was also augmented by the treatment with LPS, whereas TAP-MHC class II molecules colocalization was not affected anymore (Figure 4). These results indicate that nicotine-increased cross-presentation needs LPS-induced endosomal recruitment of TAP. 


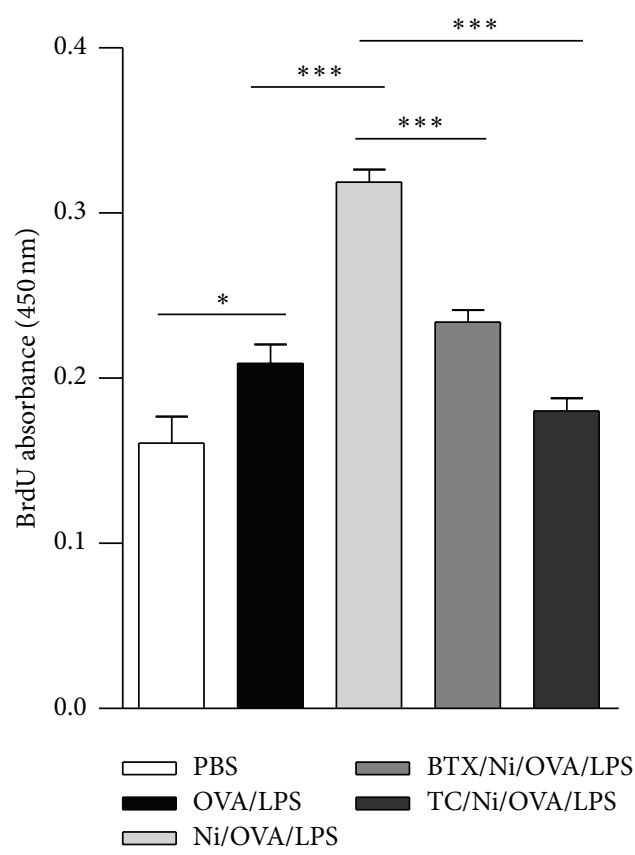

(a)

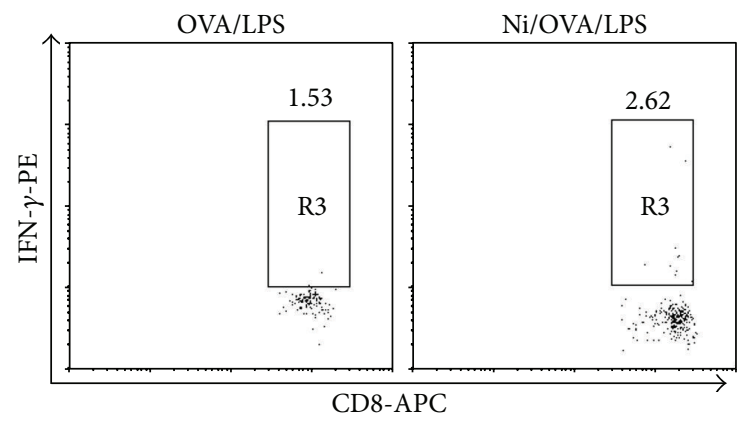

(c)

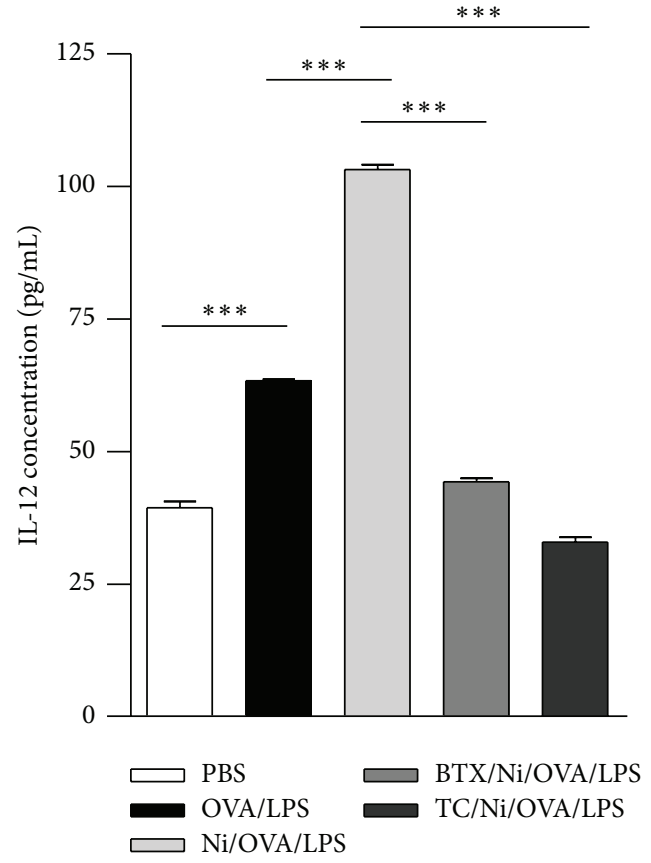

(b)

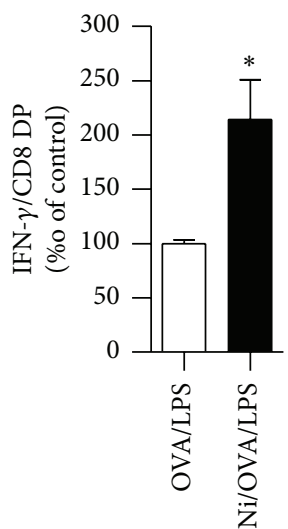

FIGURE 2: Ex vivo nicotine stimulation augments semimature DCs-mediated PBMC proliferation, promotes the release of IL-12, and increases the percentage of IFN-gamma/CD8 cells in the supernatant of cocultured DCs-PBMC. DCs derived from human PBMC with $100 \mathrm{ng} / \mathrm{mL}$ recombinant human GM-CSF and IL- 4 were conferred with $\alpha$-bungarotoxin $(2 \mu \mathrm{g} / \mathrm{mL})$ or tubocurarine chloride $\left(4 \times 10^{-5} \mathrm{~mol} / \mathrm{L}\right) 2 \mathrm{~h}$ prior to nicotine $\left(10^{-7} \mathrm{~mol} / \mathrm{L}\right) 12 \sim 15 \mathrm{~h}$ stimulation. Then, the DCs were pulsed with endotoxin-free EndoGrade-ovalbumin $(50 \mu \mathrm{g} / \mathrm{mL}) \mathrm{for} 3 \mathrm{~h}$, followed by $1 \mathrm{~h}$ LPS $(1 \mathrm{ng} / \mathrm{mL})$ stimulation. After that, the DCs were coincubated with PBMC of the same donor at a ratio of $1: 1 \mathrm{for} 5 \mathrm{~d}$ and the effect of nicotine on DCs-mediated PBMC proliferation, the IL-12 release in the supernatant, and IFN-gamma/CD8 cell percentage of cocultured DCs-PBMC were determined by BrdU cell proliferation assay (a), ELISA (b), and flow cytometry (c), respectively. Data were given as mean \pm SEM, $n=2$, one-way ANOVA with post-Newman-Keuls test. ${ }^{*} P<0.05$, and ${ }^{* * *} P<0.001$. One representative from 3 independent experiments is shown. Ni: nicotine; BTX: $\alpha$-bungarotoxin; and TC: tubocurarine chloride.

3.4. The Treatment with Nicotine Induces Akt-S6 Pathway Activation in Human Semimature DCs. It was reported that the Erk1/2-p38-JNK MAPK and PI3K-Akt pathways could be activated by the treatment with nicotine and play important roles in nicotine-augmented surface molecules expression in murine DCs $[5,6,19]$. Meanwhile, the activation of AktmTORC1 induced by nicotine promotes structural plasticity in mesencephalic dopaminergic neurons was also documented [32]. To explore the role of Akt-mTOR in nicotineincreased DCs surface molecules expression, we thereafter tested the effect of nicotine on Erk1/2-p38 MAPK and AktS6 pathway activation. Unlike traditional methods such as Western blotting, intracellular phosphoprofiling could examine cellular subpopulations in complex samples and 

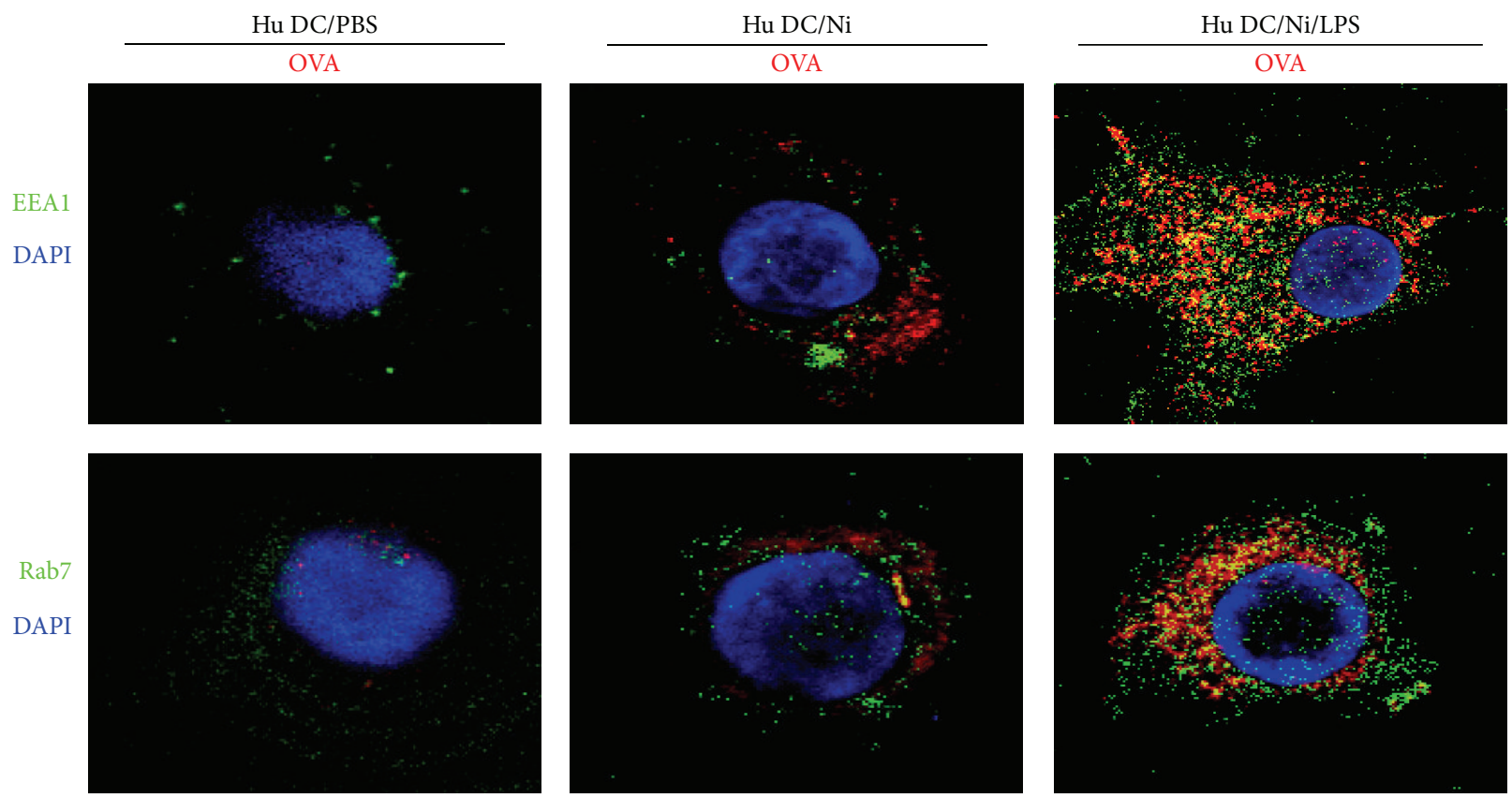

FIGURE 3: The exposure of traces of LPS increases the endosomal translocation of antigen in human semimature DCs. DCs derived from human PBMC with recombinant human GM-CSF and IL-4 were conferred with nicotine $\left(10^{-7} \mathrm{~mol} / \mathrm{L}\right) 12 \sim 15 \mathrm{~h}$ stimulation. Then, the DCs were exposed to $60 \mathrm{~min}$ endotoxin-free EndoGrade-ovalbumin $(50 \mu \mathrm{g} / \mathrm{mL})$ pulse and $20 \mathrm{~min} \mathrm{LPS}(1 \mathrm{ng} / \mathrm{mL})$ stimulation. The effect of nicotine and LPS on endosomal translocation of antigen was observed by confocal microscope analyses. One representative from 3 independent experiments is shown. Ni: nicotine.

analyze phosphoprotein signaling in rare cell subtypes [33, 34]. While the phosphorylation of Erk1/2 and p38 could be obviously augmented by nicotine stimulation (Supplementary Figure 4), the treatment with nicotine rapidly increased the phosphorylation status of Akt and S6 in the early 15 minutes, which revealed $163.5 \%$ and $191.8 \%$ increase, respectively, by flow cytometry assay (Figure 5(a)). Western blotting (Figure 5(b)) and confocal microscope analyses (Figure 5(c)) also showed that Akt-S6 pathway could be activated by the treatment with nicotine, indicating that Erk1/2-p38 and Akt-S6 pathways might be play potential roles in nicotineincreased surface molecules expression.

\subsection{Nicotine Increases Surface Molecule Expression in Human} Semimature DCs by Activating Akt-S6 Pathway. Despite the treatment with nicotine activates Akt-S6 pathway, the exact role of Akt-S6 in nicotine-increased surface molecule expression in hu-imDCs is still uncertain. We accessed the expression of surface molecules in human DCs by inhibition relevant kinases. Compared with vehicle-treated cells, the treatment with nicotine increased about $162.94 \%, 216.4 \%$, $322.4 \%, 149 \%$, and $126 \%$ expression of CD80, MHC class I, MHC class II, CD86, and 4-1BBL, respectively (Figure 6). Importantly, in contrast to the treatment with nicotine, the pretreatment with both Akt inhibitor LY294002 and Wortmannin obviously abolished nicotine's effect on these surface molecules upregulation (Figure 6). All these results indicate that the activation of Akt-S6 is involved in nicotineincreased surface molecules expression in hu-imDCs.

\section{Discussion}

In the present study, we investigated the effect of nicotine on surface molecules expression, the ability of crosspresentation, DCs-mediated PBMC priming, and activated signaling pathways by exposing hu-imDCs to nicotine stimulation. We demonstrate that the upregulation of surface molecules, the enhancement of hu-imDCs-mediated PBMC proliferation, and increased release of IL-12 in the supernatant of cocultured DCs-PBMC are $\alpha 7 \mathrm{nAchR}$-dependent. Importantly, the percentage of CD8/IFN-gamma double positive cell was increased by the treatment with ex vivo nicotine stimulation. Moreover, the phosphorylation of Akt and ribosomal protein S6 induced by nicotine stimulation play vital roles in nicotine-increased surface molecules expression. Interestingly, the endosomal translocation of internalized ovalbumin and increased TAP-OVA colocalization are also augmented by the treatment with traces of LPS.

Nicotine, a major component of cigarette smoke which promotes established tumor metastasis and increases overall mortality in cancer patients [7], is widely accepted as a risk factor for atherosclerosis [19]. Since the nAchR is mainly expressed in neuron and affects neurodegenerative disease progression [35], the effects of nicotine on promoting lung cancer development, reducing the efficacy of chemotherapeutic agents [8], and activating hypoxia-inducible factor- $1 \alpha$ expression [9] can not exclude the potential roles of nAchR in regulating the function of DCs and neuron. Recently, $\alpha 7 \mathrm{nAchR}$ has been documented to exist in murine DCs and play pivotal roles in regulating DCs' function [3-6, 

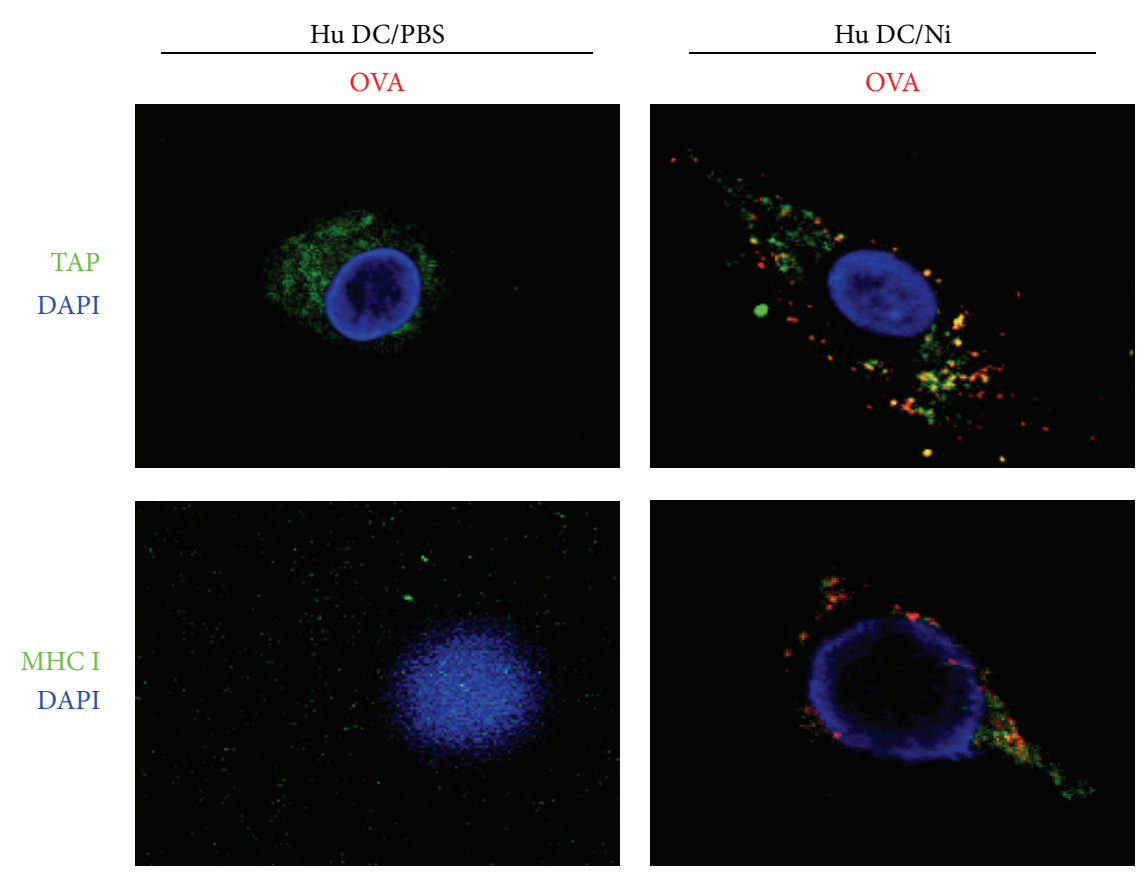

TAP
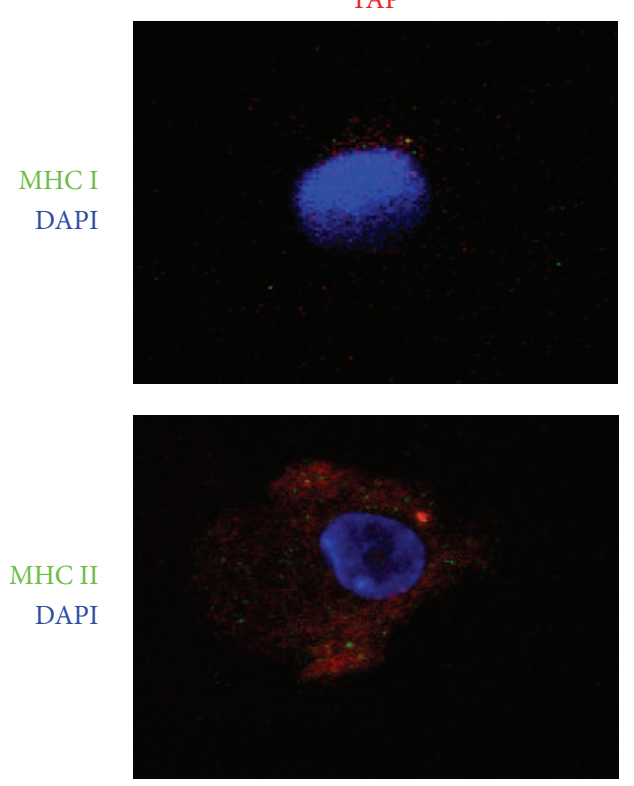

TAP
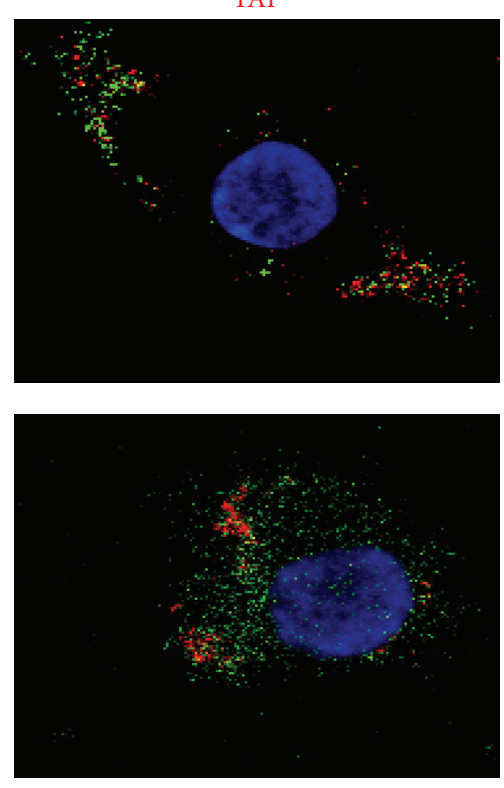
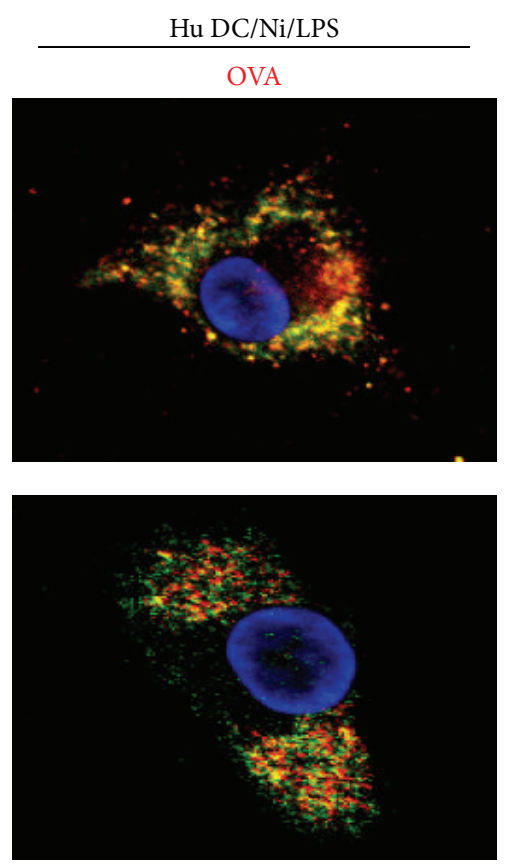

TAP
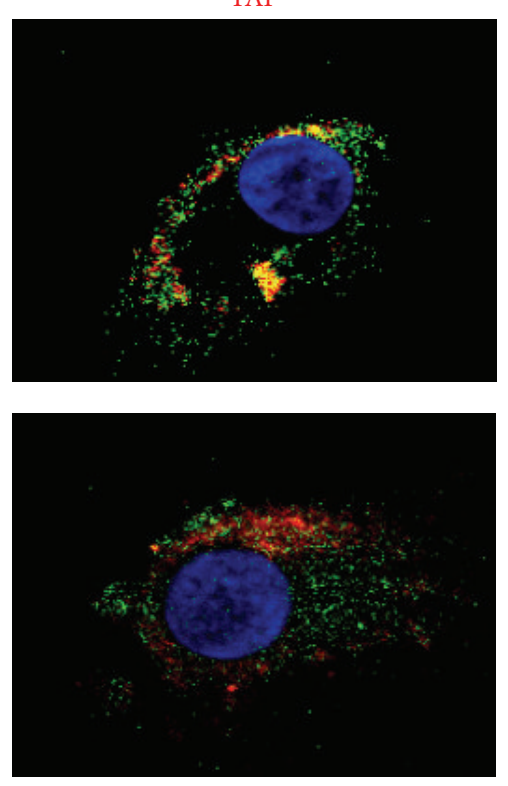

FIgURE 4: The exposure of traces of LPS increases TAP-OVA-MHC class I molecules complex formation in human semimature DCs. DCs derived from human PBMC with recombinant human GM-CSF and IL-4 were conferred with nicotine $\left(10^{-7} \mathrm{~mol} / \mathrm{L}\right) 12 \sim 15 \mathrm{~h}$ stimulation. Then, the DCs were exposed to $60 \mathrm{~min}$ endotoxin-free EndoGrade-ovalbumin $(50 \mu \mathrm{g} / \mathrm{mL})$ pulse and $20 \mathrm{~min} \mathrm{LPS}(1 \mathrm{ng} / \mathrm{mL}) \mathrm{stimulation}$. The effect of nicotine and LPS on the complex formation of TAP, OVA, and MHC class I molecules was observed by confocal microscope analyses. One representative from 3 independent experiments is shown. Ni: nicotine.

22]. In response to Th2-promoting stimuli, both mouse and human DCs generated to 6-7 days in the presence of the immune modulator nicotine (nicDCs) preferentially support the differentiation of antigen-specific IL-4-producing Th2 effector cells [15]. Furthermore, NicDCs could produce lower levels of proinflammatory cytokines when compared with DCs differentiated in the absence of nicotine $[16,17]$, indicating the modulating role of nicotine in DCs' development. Hence, the exact effect of nicotine on DCs' function is currently contradictory. Using splenic stromal cells to mimic the immune microenvironment, murine immature DCs (cultured 4 days) could induce their differentiation into a new regulatory DC subset by both stromal cell contact and stromal cell-derived transforming growth factor-beta [18]. 


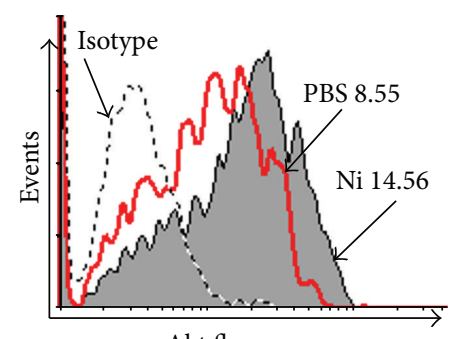

p-Akt fluorescence

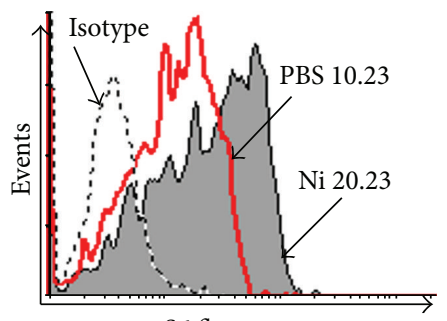

p-S6 fluorescence

(a)
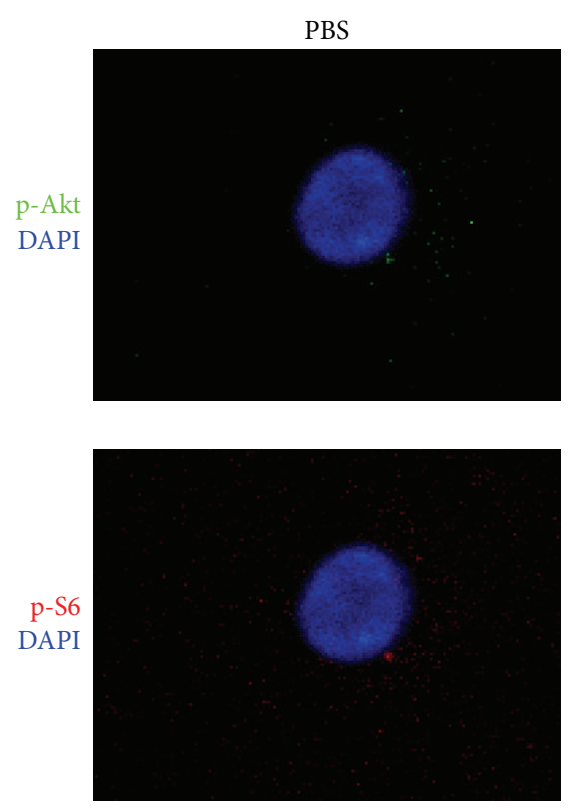

(c)
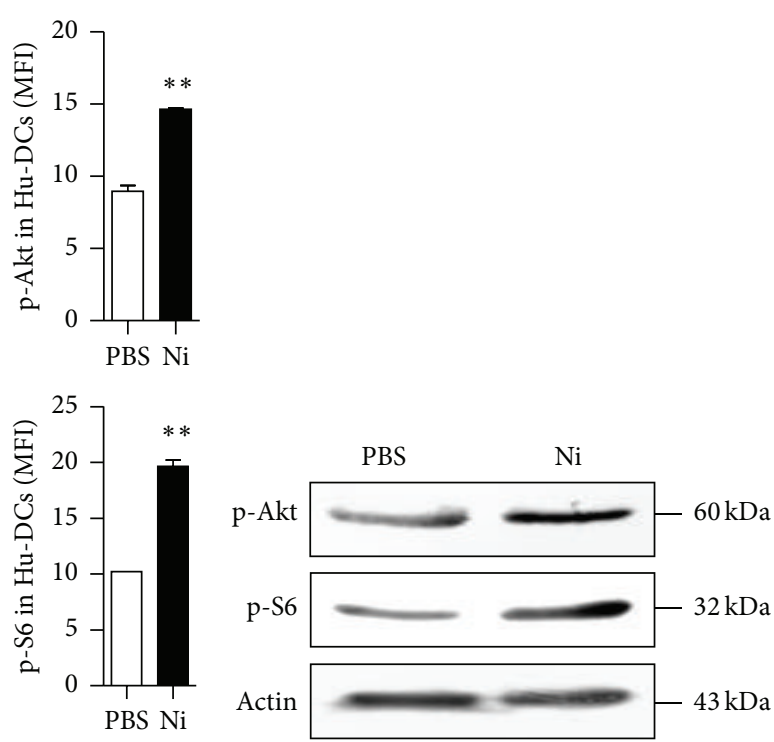

(b)
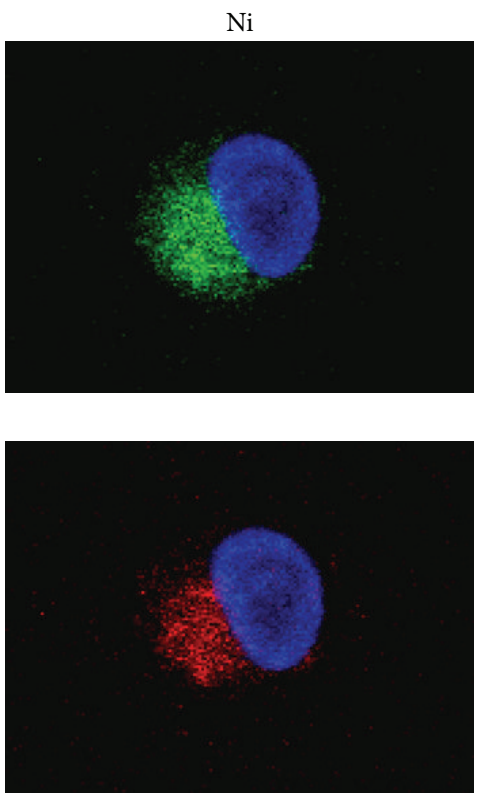

FIgURE 5: The treatment with nicotine induces Akt-S6 pathway activation in human semimature DCs. (a-c) Human PBMC-derived semimature DCs were stimulated with nicotine $\left(10^{-7} \mathrm{~mol} / \mathrm{L}\right)$ for $15 \mathrm{~min}$ and the phosphorylation of Akt and ribosomal protein S6 was determined by flow cytometry (a), Western blotting (b), and confocal microscope analyses (c), respectively. One representative from 3 independent experiments is shown. Numbers in histogram indicated mean fluorescence intensity (MFI) of test samples. Data were given as mean \pm SEM, Student $t$-test, ${ }^{* *} P<0.01$. Ni: nicotine.

In our previous studies, murine immature DCs (cultured 4 days) have been demonstrated to reveal potential antitumor effects by ex vivo nicotine stimulation [3-6]. Further studies reveal that although the treatment with nicotine and LPS upregulates surface molecules expression [20] and enables DCs to present Ags in the context of MHC I molecules, the $\mathrm{CD}^{+}$T-cell priming is refractory [36]. As the biological effect of nicotine on lymphocyte is dependent on dose of nicotine, the duration of exposure [37], and the LPS existence in experiment system [20], the controversy of nicotine's effect on DCs might be attributed to the differences of experimental design, species, duration of exposure, and especially the nicotine concentration used in these experiments. With the treatment of $200 \mathrm{mg} / \mathrm{mL}$ nicotine, Nouri-Shirazi and Guinet found that the exposure to nicotine adversely affects the dendritic cell system and compromises host response to vaccination [38]. On the other hand, the presence of nicotine $(0-200 \mu \mathrm{g} / \mathrm{mL})$ promotes the development of mouse DC precursors into a semimature phenotype and supports the differentiation of ovalbumin- (OVA-) specific naïve $\mathrm{T}$ cells 


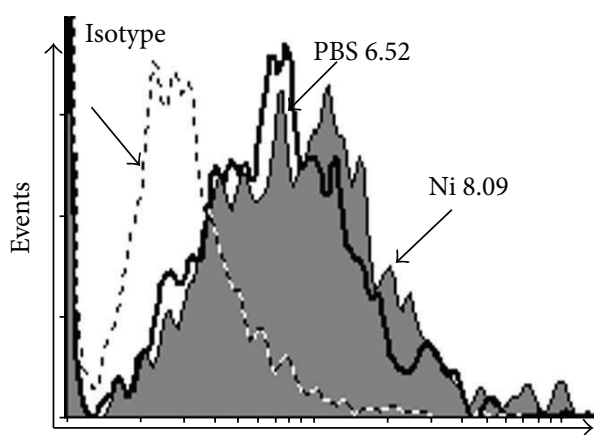

CD80 fluorescence

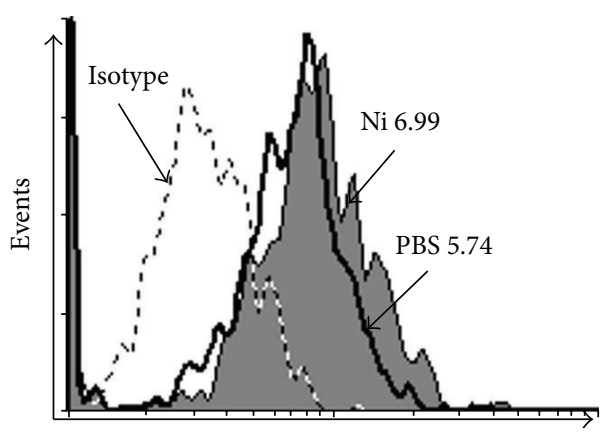

MHC I fluorescence

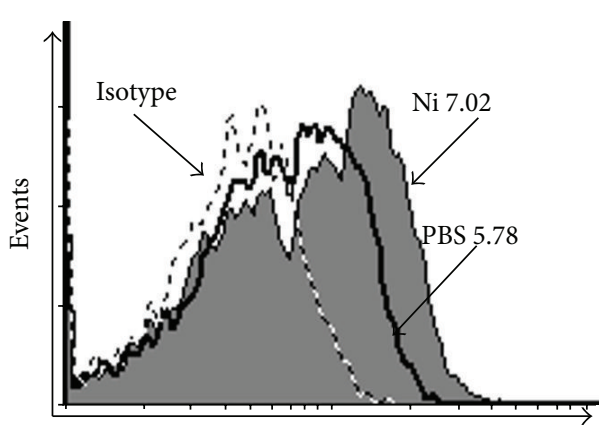

MHC II fluorescence

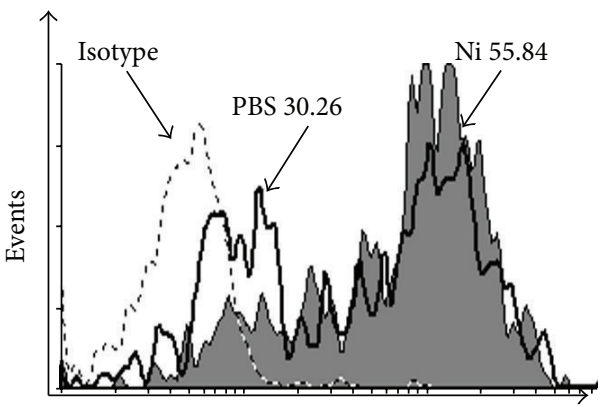

CD86 fluorescence

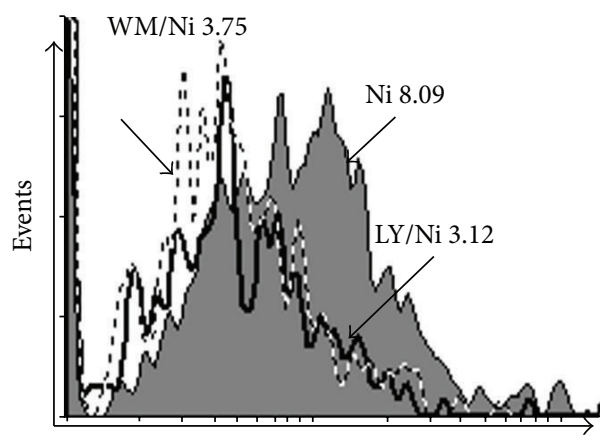

CD80 fluorescence

(a)

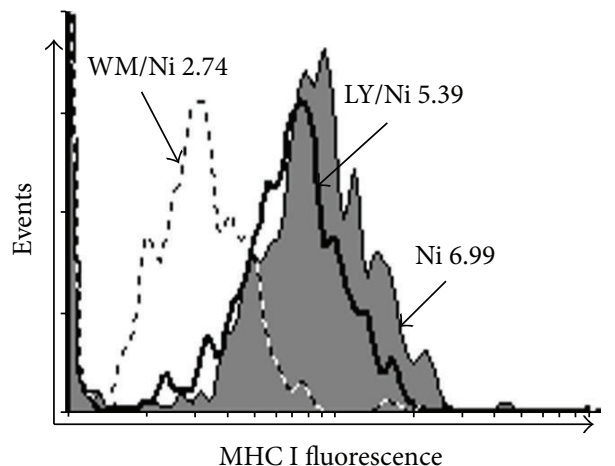

(b)
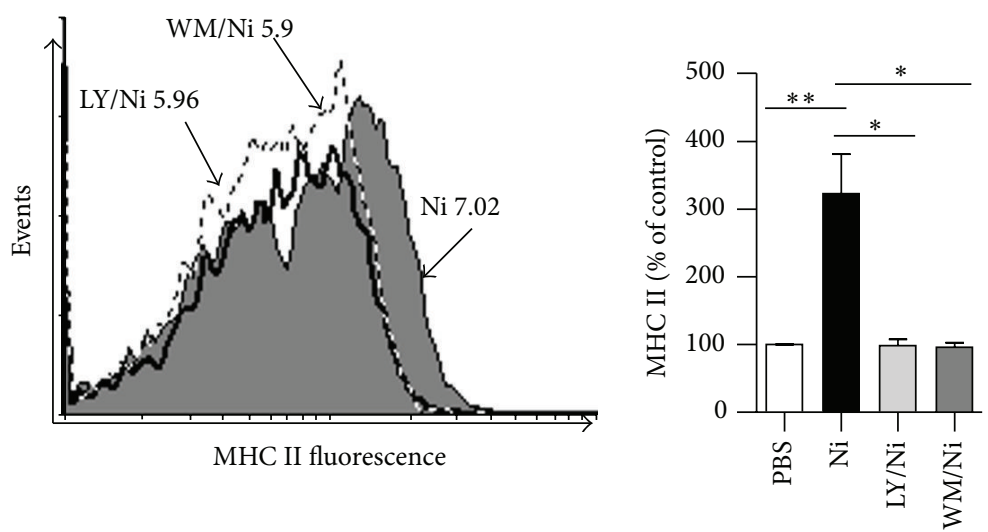

(c)
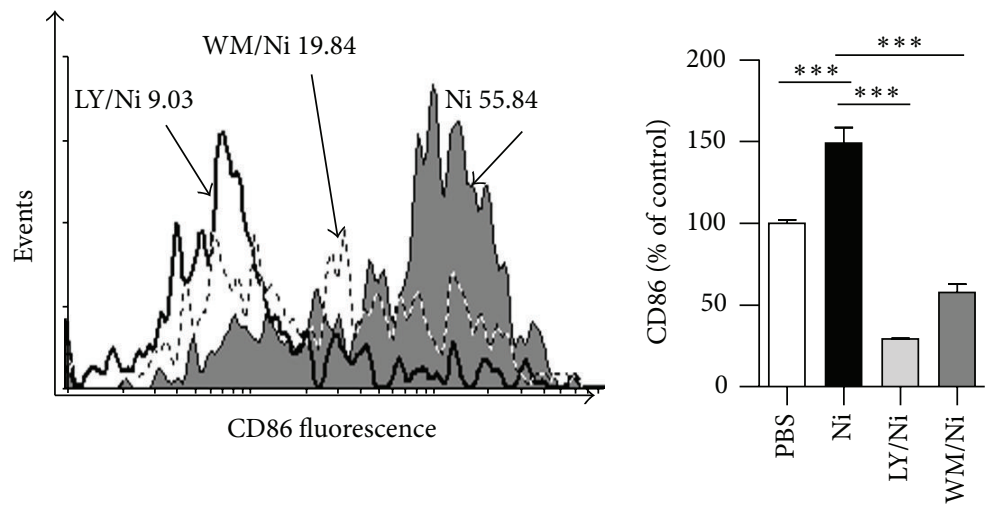

(d) 


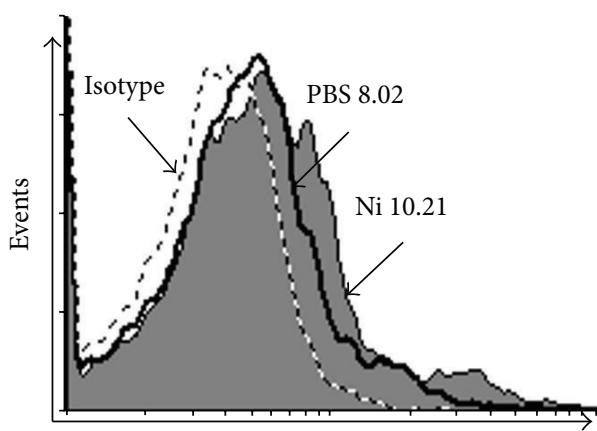

4-1BBL fluorescence

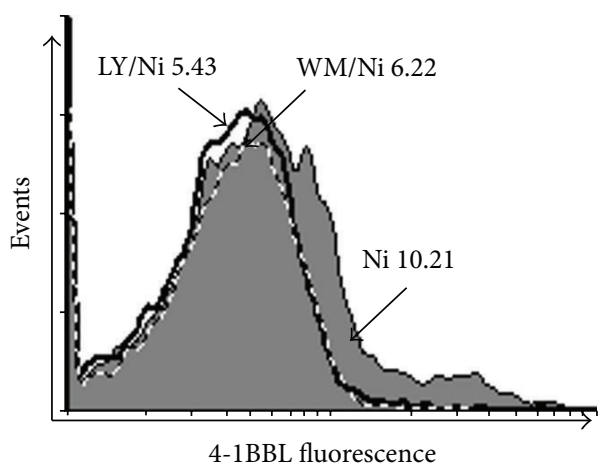

(e)

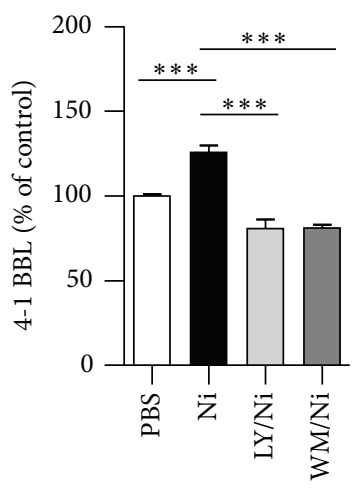

FIGURE 6: Nicotine increases surface molecule expression in human semimature DCs by activating Akt-S6 pathway. (a-e) DCs derived from human PBMC with human GM-CSF and IL-4 were conferred with LY294002 $(10 \mu \mathrm{mol} / \mathrm{L})$ or Wortmannin $(10 \mu \mathrm{mol} / \mathrm{L}) 2 \mathrm{~h}$ prior to $12 \sim 15 \mathrm{~h}$ nicotine $\left(10^{-7} \mathrm{~mol} / \mathrm{L}\right)$ stimulation. The effect of Akt inhibition on nicotine-increased expression of CD80 (a), MHC class I (b), MHC class II (c), CD86 (d), and 4-1BBL (e) was determined by flow cytometry. Numbers in histogram indicated mean fluorescence intensity (MFI) of test samples. Data were given as mean $\pm \mathrm{SEM}$, one-way ANOVA with post Newman-Keuls test. ${ }^{*} P<0.05,{ }^{* *} P<0.01$, and ${ }^{* * *} P<0.001$. One representative from 3 independent experiments is shown. Ni: nicotine; LY: LY294002; WM (Wort): Wortmannin.

into effector memory cells [21]. In our systematic studies, $10^{-7} \mathrm{~mol} / \mathrm{L}$ nicotine $(16.5 \mathrm{ng} / \mathrm{mL})$ was used as $\alpha 7 \mathrm{nAchR}$ agonist to stimulate human or murine semimature DCs (cultured 4 days). Hence, lower dose nicotine stimulation $(16.5 \mathrm{ng} / \mathrm{mL})$ and DCs with a semimature phenotype were the specific conditions for achieving increased DCs vaccination. It does not implicate that nicotine itself has the similar biological effect on cancer growth.

Nicotine increasing mouse immature DCs-mediated cross priming could be abolished by $\alpha 7 \mathrm{nAchR}$ specific antagonist $\alpha$-bungarotoxin and nonspecific antagonist tubocurarine chloride [3-6], indicating the vital role of $\alpha 7 \mathrm{nAchR}$ in nicotine-augmented murine DCs' function. In the present study, hu-imDCs were pretreated with $\alpha$-bungarotoxin or tubocurarine chloride prior to ex vivo nicotine stimulation to investigate the role of $\alpha 7 \mathrm{nAchR}$ in nicotine-augmented huimDCs' function. Consistent with the finding of murine DCs, not only the upregulation of surface molecules (Figure 1) but also DCs-mediated PBMC priming (Figure 2) was abrogated by the blockage of $\alpha 7 \mathrm{nAchR}$. These data indicate that nicotine-increased DCs-dependent PBMC proliferation and surface molecules upregulation are also $\alpha 7 \mathrm{nAchR}$ dependent.

Antigens internalized by DCs via fluid phage pinocytosis or scavenger receptor-mediated endocytosis are rapidly targeted toward lysosomal structures, where they are degraded instantly and processed for presentation on MHC II molecules [39]. Meanwhile, antigens, which are internalized by the mannose receptor, are routed into endosome subpopulation. Antigens of endosome compartment are protected from lysosomal degradation and are processed exlusively for cross-presentation [12]. Recently, endotoxin was found to increase recruitment of TAP toward antigen-containing endosomes and enable the retranslocation of proteasomederived peptides into the same endosome subset [12, 27, 28]. In this study, compared with endotoxin-free ovalbumin loading, the pulse of endotoxin-free ovalbumin with traces of LPS not only increased the endosomal translocation of antigen (Figure 3) but also augmented the formation of OVA-TAP-MHC class I complex (Figure 4), indicating that nicotine-enhanced hu-imDCs cross-presentation might also attribute to LPS-increased endosomal protection from lysosomal degradation and the endosomal recruitment of TAP.

Akt, which could be phosphorylated within the activation loop at threonine 308 and the C-terminus at serine 473, promotes cell survival by inhibiting proapoptotic function of Bad [40]. We have demonstrated that nicotine activates PI3KAkt pathway and upregulates CD80 molecules expression in murine DCs $[5,6]$. Jossin and Goffinet's study also showed that PI3K-Akt signal controls cortical development and regulates dendritic growth [41]. In the present study, Akt pathway was found to play vital role in nicotine-increased surface molecules expression in hu-imDCs (Figure 6). Ribosomal protein S6, which plays a role in regulating the translation of RNAs and thus controlling the growth and proliferation of cells [42], was efficient phosphorylated by the treatment with nicotine (Figure 5). Although mTORC1 was involved in nicotine-induced structural plasticity in mesencephalic dopaminergic neurons [32], the phosphorylation of S6 ribosomal protein was reported to upregulate ribosomal translocation of RNA species coding for other ribosomal proteins, peptide elongation factors [43]. Hence, the exact role of S6 ribosomal protein in nicotine-regulated surface molecules expression in human DCs still needs further investigation.

In conclusion, all the data presented here indicate that nicotine upregulating surface molecules and enhancing DCsmediated PBMC proliferation and the release of IL-12 are $\alpha 7$ nAchR-dependent. Nicotine-enhanced hu-imDCs crosspresentation attributes to LPS-increased endosomal translocation of internalized ovalbumin and the endosomal recruitment of TAP. 


\section{Abbreviations}

DCs: Dendritic cells

nAChR: Nicotinic acetylcholine receptor

S6: $\quad$ Ribosomal protein S6

PI3K: Phosphatidylinositol-3-kinase

Ni: $\quad$ Nicotine

MLRs: Mixed lymphocyte reactions

LPS: Lipopolysaccharide

PBMC: Peripheral blood monouclear cells

EEA1: Early endosome antigen 1

TAP: Transporter associated with antigen processing

Rab1: Ras-Related superfamily of guanine nucleotide binding proteins 1 .

\section{Conflict of Interests}

The authors declare that they have no conflict of interests.

\section{Authors' Contribution}

Yan Yan Wang, Yi Wen Yang, and Xiang You contributed equally to this paper. Feng Guang Gao designed the research and copyedited the paper. Yan Yan Wang and Xiang You contributed to FACS, MLR, ELISA, and confocal microscope observation. Chun Fang Hu contributed to Western blot. Xiao Qian Deng and Yi Nan Wang contributed to MLR and ELISA. Cong Zhu, Jun Yao Wang, and Jiao Jiao Gu contributed to human DCs induction. Yi Wen Yang contributed to flow cytometry and confocal microscope observation. Qing Li wrote the paper.

\section{Acknowledgments}

This work was supported by Grants from the State Key Laboratory of Oncogenes and Related Genes (no. 90-14-05), Grants from the National Natural Science Foundation of China (no. 81273203; no. 81201275), and Grants from Natural Science Foundation of Fujian Province of China (nos. 2015J01353, 2013J05124).

\section{References}

[1] J. D. Bassett, T. C. Yang, D. Bernard et al., "CD8 ${ }^{+}$T-cell expansion and maintenance after recombinant adenovirus immunization rely upon cooperation between hematopoietic and nonhematopoietic antigen-presenting cells," Blood, vol. 117, no. 4, pp. 1146-1155, 2011.

[2] N.-L. L. Pham, L. L. Pewe, C. J. Fleenor et al., "Exploiting crosspriming to generate protective CD8 T-cell immunity rapidly," Proceedings of the National Academy of Sciences of the United States of America, vol. 107, no. 27, pp. 12198-12203, 2010.

[3] G. G. Feng, F. W. Da, and R. G. Jian, "Ex vivo nicotine stimulation augments the efficacy of therapeutic bone marrowderived dendritic cell vaccination," Clinical Cancer Research, vol. 13, no. 12, pp. 3706-3712, 2007.

[4] F. G. Gao, H. T. Li, Z. J. Li, and J. R. Gu, "Nicotine stimulated dendritic cells could achieve anti-tumor effects in mouse lung and liver cancer," Journal of Clinical Immunology, vol. 31, no. 1, pp. 80-88, 2011.

[5] H. J. Jin, H. T. Li, H. X. Sui et al., "Nicotine stimulated bone marrow-derived dendritic cells could augment HBV specific CTL priming by activating PI3K-Akt pathway," Immunology Letters, vol. 146, no. 1-2, pp. 40-49, 2012.

[6] H. J. Jin, H. X. Sui, Y. N. Wang, and F. G. Gao, "Nicotine upregulated 4-1BBL expression by activating Mek-PI3K pathway augments the efficacy of bone marrow-derived dendritic cell vaccination," Journal of Clinical Immunology, vol. 33, no. 1, pp. 246-254, 2013.

[7] R. J. Rangani, M. A. Upadhya, K. T. Nakhate, D. M. Kokare, and N. K. Subhedar, "Nicotine evoked improvement in learning and memory is mediated through NPY Y1 receptors in rat model of Alzheimer's disease," Peptides, vol. 33, no. 2, pp. 317-328, 2012.

[8] S. Nikfar, S. Ehteshami-Ashar, R. Rahimi, and M. Abdollahi, "Systematic review and meta-analysis of the efficacy and tolerability of nicotine preparations in active ulcerative colitis," Clinical Therapeutics, vol. 32, no. 14, pp. 2304-2315, 2010.

[9] M. Orth, B. Amann, M. M. Robertson, and J. C. Rothwell, "Excitability of motor cortex inhibitory circuits in Tourette syndrome before and after single dose nicotine," Brain, vol. 128, no. 6, pp. 1292-1300, 2005.

[10] C. Kreer, J. Rauen, M. Zehner, and S. Burgdorf, "Crosspresentation: how to get there-or how to get the ER," Frontiers in Immunology, vol. 2, article 87, 2012.

[11] S. Burgdorf, V. Schuette, V. Semmling et al., "Steady-state cross-presentation of OVA is mannose receptor-dependent but inhibitable by collagen fragments," Proceedings of the National Academy of Sciences of the United States of America, vol. 107, no. 13, pp. E48-E49, 2010.

[12] S. Burgdorf, C. Schölz, A. Kautz, R. Tampé, and C. Kurts, "Spatial and mechanistic separation of cross-presentation and endogenous antigen presentation," Nature Immunology, vol. 9, no. 5, pp. 558-566, 2008.

[13] M. Zehner, A. I. Chasan, V. Schuette et al., "Mannose receptor polyubiquitination regulates endosomal recruitment of p97 and cytosolic antigen translocation for cross-presentation," Proceedings of the National Academy of Sciences of the United States of America, vol. 108, no. 24, pp. 9933-9938, 2011.

[14] M. Zehner and S. Burgdorf, "Regulation of antigen transport into the cytosol for cross-presentation by ubiquitination of the mannose receptor," Molecular Immunology, vol. 55, no. 2, pp. 146-148, 2013.

[15] M. Nouri-Shirazi, C. Kahlden, P. Nishino, and E. Guinet, "Nicotine exposure alters the mRNA expression of Notch ligands in dendritic cells and their response to Th1-/Th2-promoting stimuli," Scandinavian Journal of Immunology, vol. 81, no. 2, pp. 110120, 2015.

[16] M. Yanagita, K. Mori, R. Kobayashi et al., "Immunomodulation of dendritic cells differentiated in the presence of nicotine with lipopolysaccharide from Porphyromonas gingivalis," European Journal of Oral Sciences, vol. 120, no. 5, pp. 408-414, 2012.

[17] M. Yanagita, R. Kobayashi, Y. Kojima, K. Mori, and S. Murakami, "Nicotine modulates the immunological function of dendritic cells through peroxisome proliferator-activated receptor- $\gamma$ upregulation," Cellular Immunology, vol. 274, no. 12, pp. 26-33, 2012.

[18] M. Zhang, H. Tang, Z. Guo et al., "Splenic stroma drives mature dendritic cells to differentiate into regulatory dendritic cells," Nature Immunology, vol. 5, no. 11, pp. 1124-1133, 2004. 
[19] A. Aicher, C. Heeschen, M. Mohaupt, J. P. Cooke, A. M. Zeiher, and S. Dimmeler, "Nicotine strongly activates dendritic cellmediated adaptive immunity: potential role for progression of atherosclerotic lesions," Circulation, vol. 107, no. 4, pp. 604-611, 2003.

[20] S. X. Hu, H. X. Sui, H. J. Jin et al., "Lipopolysaccharide and dose of nicotine determine the effects of nicotine on murine bone marrow-derived dendritic cells," Molecular Medicine Reports, vol. 5, no. 4, pp. 1005-1010, 2012.

[21] M. Nouri-Shirazi, R. Tinajero, and E. Guinet, "Nicotine alters the biological activities of developing mouse bone marrowderived dendritic cells (DCs)," Immunology Letters, vol. 109, no. 2, pp. 155-164, 2007.

[22] M. Grujic, C. Bartholdy, M. Remy, D. D. Pinschewer, J. P. Christensen, and A. R. Thomsen, "The role of CD80/CD86 in generation and maintenance of functional virus-specific $\mathrm{CD} 8^{+}$ $\mathrm{T}$ cells in mice infected with lymphocytic choriomeningitis virus," Journal of Immunology, vol. 185, no. 3, pp. 1730-1743, 2010.

[23] Z.-Y. Lu, M. Condomines, K. Tarte et al., "B7-1 and 4-1BB ligand expression on a myeloma cell line makes it possible to expand autologous tumor-specific cytotoxic T cells in vitro," Experimental Hematology, vol. 35, no. 3, pp. 443-453, 2007.

[24] C. Wu, H. Guo, Y. Wang, Y. Gao, Z. Zhu, and Z. Du, "Extracellular domain of human 4-1BBL enhanced the function of cytotoxic T-lymphocyte induced by dendritic cell," Cellular Immunology, vol. 271, no. 1, pp. 118-123, 2011.

[25] S. Kanagavelu, J. M. Termini, S. Gupta et al., "HIV-1 adenoviral vector vaccines expressing multi-trimeric BAFF and 4-1BBL enhance T cell mediated anti-viral immunity," PLOS ONE, vol. 9, no. 2, Article ID e90100, 2014.

[26] C. Wang, T. Wen, J.-P. Routy, N. F. Bernard, R. P. Sekaly, and T. H. Watts, "4-1BBL induces TNF receptor-associated factor 1-dependent Bim modulation in human $\mathrm{T}$ cells and is a critical component in the costimulation-dependent rescue of functionally impaired HIV-specific CD8 T cells," Journal of Immunology, vol. 179, no. 12, pp. 8252-8263, 2007.

[27] J. M. Blander and R. Medzhitov, "Toll-dependent selection of microbial antigens for presentation by dendritic cells," Nature, vol. 440, no. 7085, pp. 808-812, 2006.

[28] B. C. Gil-Torregrosa, A. M. Lennon-Duménil, B. Kessler et al., "Control of cross-presentation during dendritic cell maturation," European Journal of Immunology, vol. 34, no. 2, pp. 398407, 2004.

[29] S. Gupta, R. Boppana, G. C. Mishra, B. Saha, and D. Mitra, "Interleukin-12 is necessary for the priming of $\mathrm{CD}_{4}{ }^{+} \mathrm{T}$ cells required during the elicitation of HIV-1 gp120-specific cytotoxic T-lymphocyte function," Immunology, vol. 124, no. 4, pp. 553561, 2008.

[30] T. Hilmenyuk, I. Bellinghausen, B. Heydenreich et al., "Effects of glycation of the model food allergen ovalbumin on antigen uptake and presentation by human dendritic cells," Immunology, vol. 129, no. 3, pp. 437-445, 2010.

[31] S. B. Bazan, G. Geginat, T. Breinig, M. J. Schmitt, and F. Breinig, "Uptake of various yeast genera by antigen-presenting cells and influence of subcellular antigen localization on the activation of ovalbumin-specific CD8 T lymphocytes," Vaccine, vol. 29, no. 45, pp. 8165-8173, 2011.

[32] G. Collo, F. Bono, L. Cavalleri et al., "Nicotine-induced structural plasticity in mesencephalic dopaminergic neurons is mediated by dopamine D3 receptors and Akt-mTORC1 signaling," Molecular Pharmacology, vol. 83, no. 6, pp. 1176-1189, 2013.

[33] K. Blatt, H. Herrmann, I. Mirkina et al., "The PI3-kinase/ mTOR-targeting drug NVP-BEZ235 inhibits growth and IgEdependent activation of human mast cells and basophils," PLoS ONE, vol. 7, no. 1, Article ID e29925, 2012.

[34] L. van de Laar, A. van den Bosch, A. Boonstra et al., "PI3K-PKB hyperactivation augments human plasmacytoid dendritic cell development and function," Blood, vol. 120, no. 25, pp. 49824991, 2012.

[35] M. Q. Xue, X. X. Liu, Y. L. Zhang, and F. G. Gao, "Nicotine exerts neuroprotective effects against $\beta$-amyloid-induced neurotoxicity in SH-SY5Y cells through the Erk1/2-p38-JNK-dependent signaling pathway," International Journal of Molecular Medicine, vol. 33, no. 4, pp. 925-933, 2014.

[36] J. T. Tan, J. K. Whitmire, R. Ahmed, T. C. Pearson, and C. P. Larsen, " $4-1 \mathrm{BB}$ ligand, a member of the TNF family, is important for the generation of antiviral CD8 T cell responses," The Journal of Immunology, vol. 163, no. 9, pp. 4859-4868, 1999.

[37] S. T. Hanna, "Nicotine effect on cardiovascular system and ion channels," Journal of Cardiovascular Pharmacology, vol. 47, no. 3, pp. 348-358, 2006.

[38] M. Nouri-Shirazi and E. Guinet, "Exposure to nicotine adversely affects the dendritic cell system and compromises host response to vaccination," The Journal of Immunology, vol. 188, no. 5, pp. 2359-2370, 2012.

[39] S. Burgdorf, A. Kautz, V. Böhnert, P. A. Knolle, and C. Kurts, "Distinct pathways of antigen uptake and intracellular routing in CD4 and CD8 T cell activation," Science, vol. 316, no. 5824, pp. 612-616, 2007.

[40] Y. Li, M. Zeng, W. Chen et al., "Dexmedetomidine reduces isoflurane-induced neuroapoptosis partly by preserving PI3K/ Akt pathway in the hippocampus of neonatal rats," PLoS ONE, vol. 9, no. 4, Article ID e93639, 2014.

[41] Y. Jossin and A. M. Goffinet, "Reelin signals through phosphatidylinositol 3-kinase and Akt to control cortical development and through mTor to regulate dendritic growth," Molecular and Cellular Biology, vol. 27, no. 20, pp. 7113-7124, 2007.

[42] K. Höland, D. Boller, C. Hagel et al., "Targeting class Ia PI3K isoforms selectively impairs cell growth, survival, and migration in glioblastoma," PLoS ONE, vol. 9, no. 4, Article ID e94132, 2014.

[43] T. Wang, T. Kusudo, T. Takeuchi et al., "Evodiamine inhibits insulin-stimulated mTOR-S6K activation and IRS1 serine phosphorylation in adipocytes and improves glucose tolerance in obese/diabetic mice," PLoS ONE, vol. 8, no. 12, Article ID e83264, 2013. 


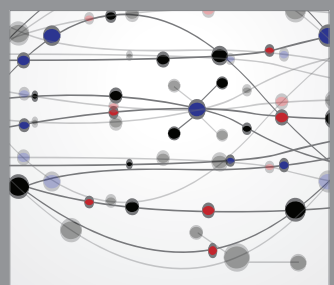

The Scientific World Journal
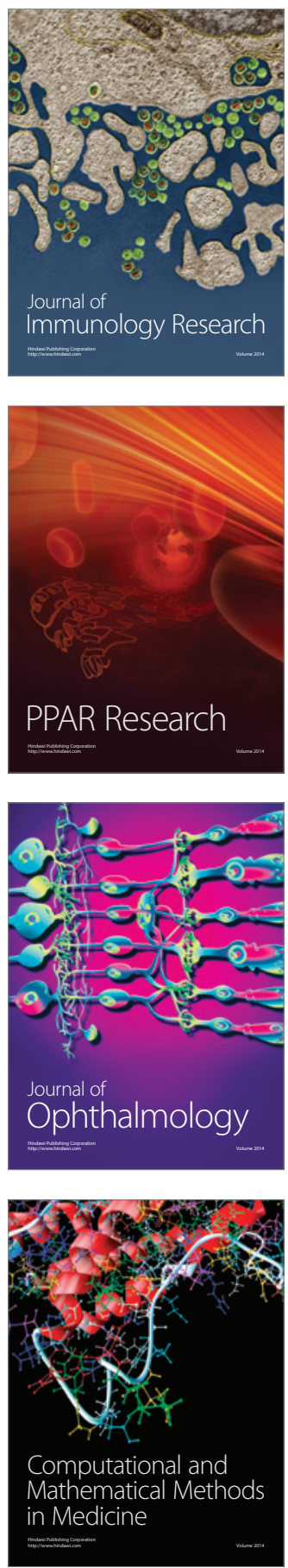

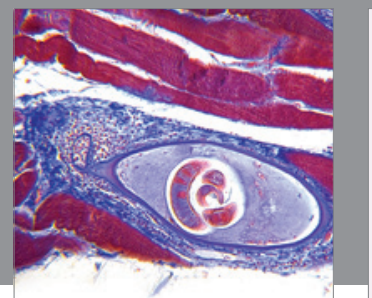

Gastroenterology

Research and Practice
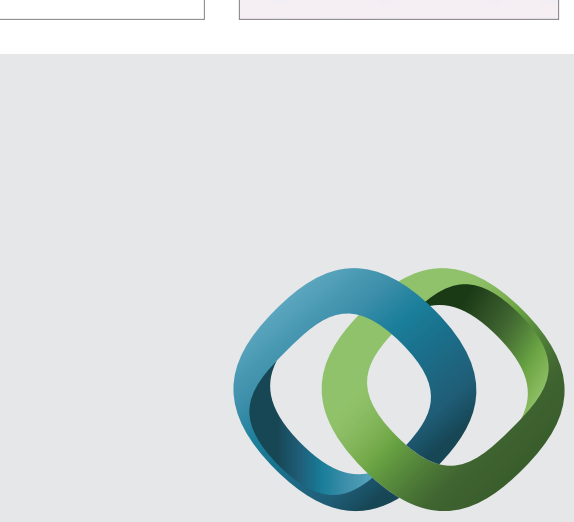

\section{Hindawi}

Submit your manuscripts at

http://www.hindawi.com
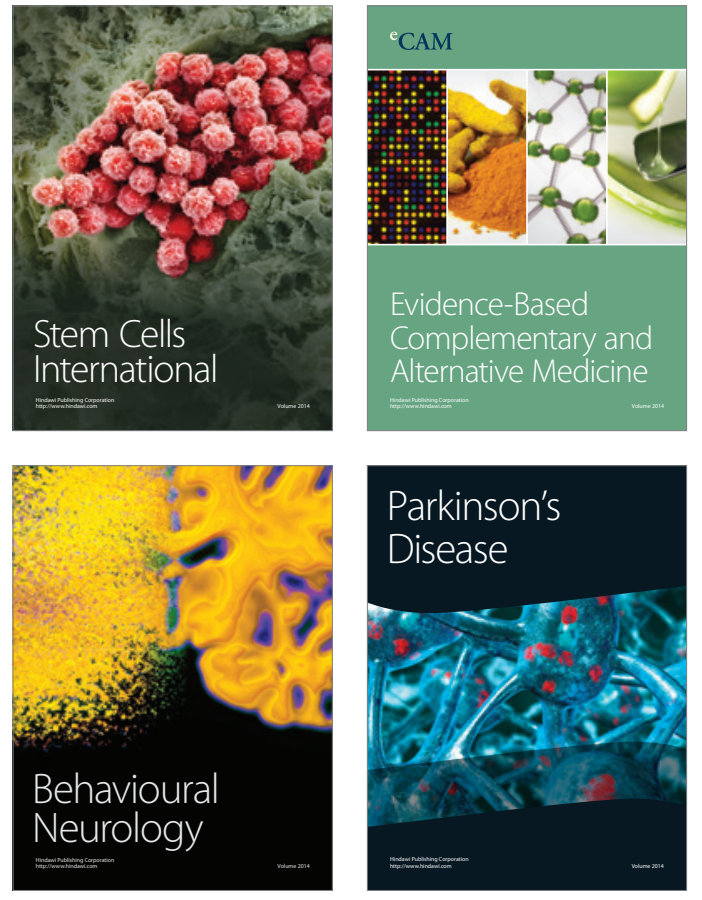
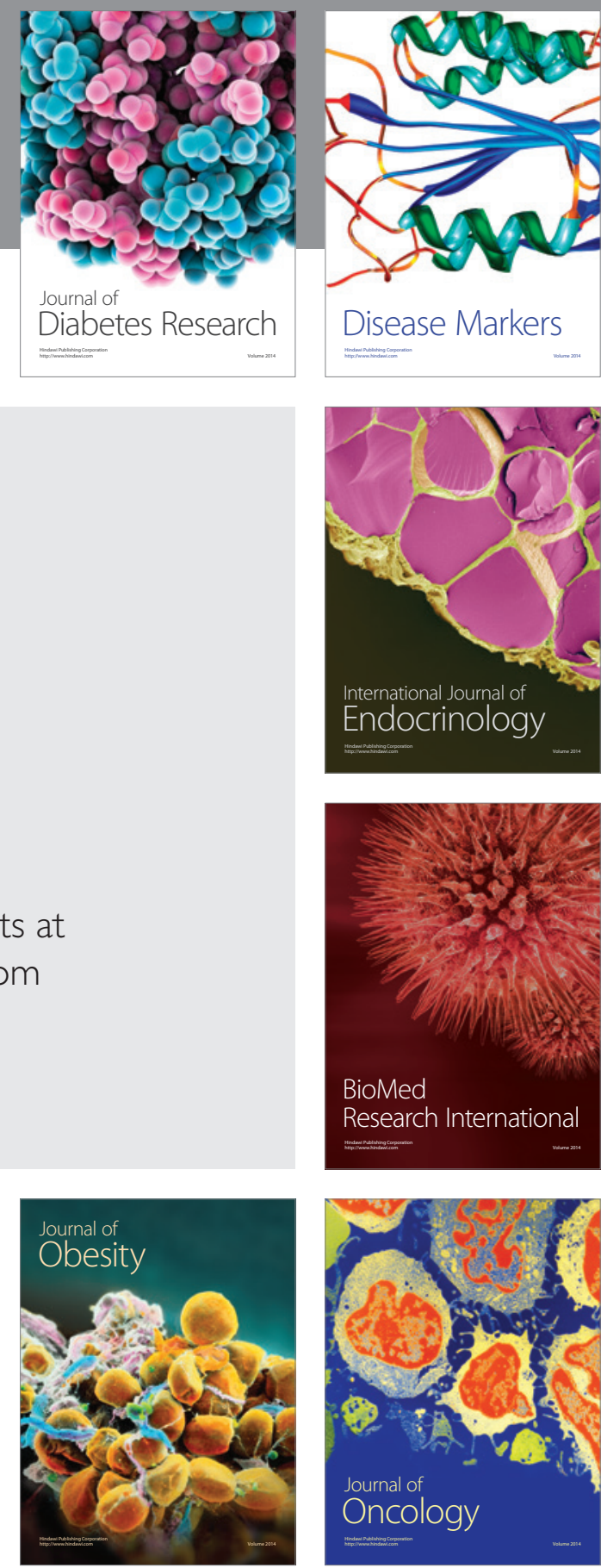

Disease Markers
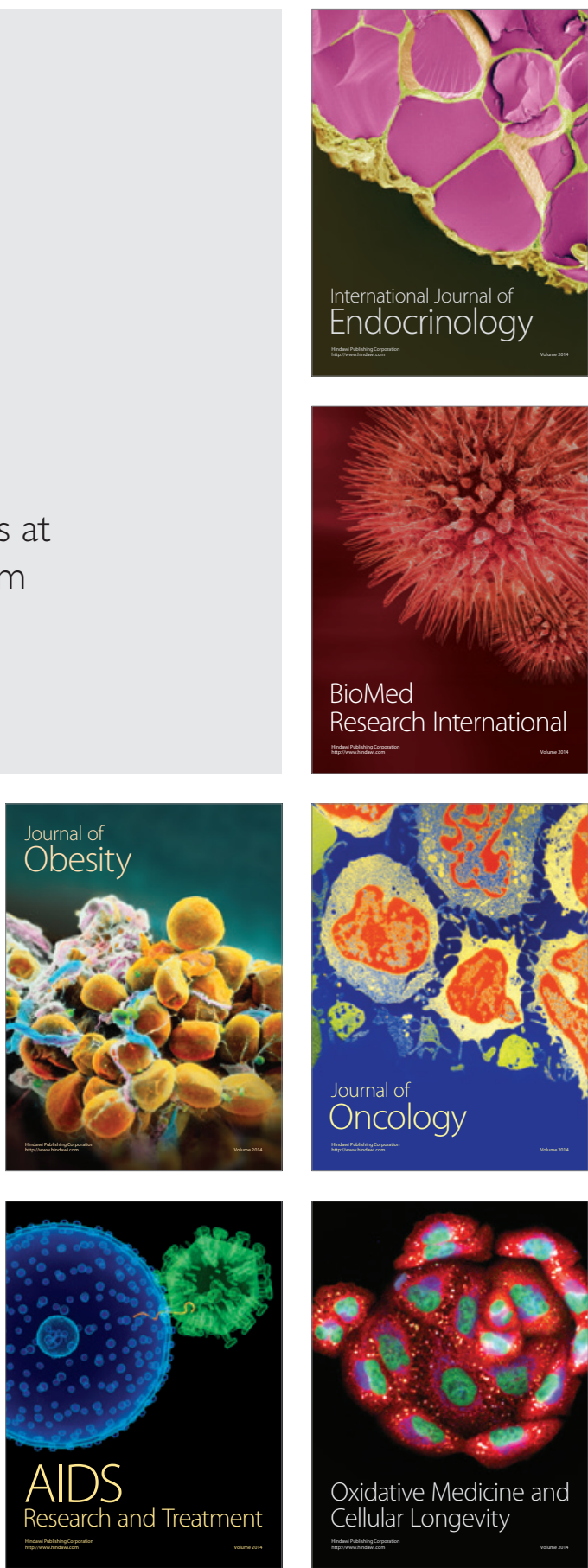